\title{
Probing Planck-scale physics with Extragalactic Sources?
}

\author{
Y. Jack Ng, W. A. Christiansen, and H. van Dam \\ Department of Physics and Astronomy, University of North Carolina, Chapel Hill, NC 27599 \\ yjng@physics.unc.edu, wayne@astro.unc.edu
}

\begin{abstract}
At Planck-scale, spacetime is "foamy" due to quantum fluctuations predicted by quantum gravity. Here we consider the possibility of using spacetime foam-induced phase incoherence of light from distant galaxies and gamma-ray bursters to probe Planck-scale physics. In particular, we critically examine the cumulative effects of spacetime fluctuations over a huge distance. Unfortunately, our analysis shows that they are far below what is required in this approach to shed light on the foaminess of spacetime.
\end{abstract}

Subject headings: Planck-scale physics, quantum foam-induced phase incoherence of light, gamma-ray bursts, distant galaxies

It is generally believed that quantum gravity, the synthesis of quantum mechanics and general relativity, predicts that spacetime becomes "foamy" or "fuzzy" at the Planck scale given by the Planck time $t_{P}=\left(\hbar G / c^{5}\right)^{1 / 2} \sim 10^{-44} s$, Planck length $l_{P}=c t_{P} \sim 10^{-33} \mathrm{~cm}$, and Planck energy $E_{P}=\hbar / t_{P} \sim 10^{28} \mathrm{eV}$. The fuzziness of spacetime leads to uncertainties in distance $(l)$ measurements whose absolute value is given by $\delta l \gtrsim l_{P}\left(l / l_{P}\right)^{1-\alpha}$ (similar uncertainties for time measurements) and uncertainties in energy $(E)$ measurements given by $\delta E \gtrsim E\left(E / E_{P}\right)^{\alpha}$ (again $\delta E$ is an absolute value). Similar uncertainties for momentum measurements also obtain.( $\mathrm{Ng} \&$ van Dam 1994) The parameter, $\alpha \sim 1$, specifies different quantum gravity models. The standard choice(Misner et al 1973) of $\alpha$ is $\alpha=1$; the choice of $\alpha=2 / 3$ appears( $\mathrm{Ng} \&$ van Dam 2000; Ng 2001) to be consistent with the holographic principle('tHooft 1993; Susskind 1995) and black hole physics; $\alpha=1 / 2$ corresponds to the random-walk model found in the literature(Amelino-Camelia 1994).

The ultra-high energy cosmic ray threshold anomalies (see, e.g., Ref. (Amelino-Camelia \& Piran 2001; Ng et al. 2001; Amelino-Camelia et al. 2002; Aloisio et al. 2002) and references therein) may have given us some tentalizing hints of Planckian fluctuations. But so far we lack direct experimental evidence.

Recently Lieu and Hillman(Lieu \& Hillman 2002, 2003) and then Ragazzoni, Turatto, and Gaessler (Ragazzoni et al. 2003) proposed a technique that has hitherto been overlooked to directly test Planck scale fluctuations. They argued that these fluctuations can cumulatively lead to a complete loss of phase coherence for radiation that has propagated a sufficiently large distance. As a result of this inferred phase scrambling attributed to Planck scale uncertainties (spacetime foam) these authors concluded that distant compact radiation sources should not produce the normal interference patterns (e.g., Airy rings) which are often observed. In this Letter, we critically examine their very interesting idea and extend their proposal to include the use of gamma-ray burst interferometry.

Since the cumulative effect of quantum foam on the phase coherence of light from extragalactic sources figures crucially in the method, we begin by establishing how large the cumulative effects are, especially for the three quantum gravity models mentioned above.

Consider a distance $L$ (the reader may anticipate this to denote the distance between the extragalactic source and the telescope). Let us di- 
vide it into $L / \lambda$ equal parts each of which has length $\lambda$ (the reader may anticipate this to denote the wavelength of the observed light from the distant source). We already know that the absolute value of the uncertainty in distance $L$ is given by $\delta L \sim l_{P}\left(L / l_{P}\right)^{1-\alpha}$.

But let us calculate it again by starting with $\delta \lambda$ for each part and then adding up the contributions to $\delta L$ from the $L / \lambda$ parts in $L$. In doing so we will find out how large the cumulative effects from the various $\delta \lambda$ 's are. To gain insight into the process, let us first consider the $\alpha=1 / 2$ randomwalk model of quantum gravity. In this model, the typical quantum fluctuation $\delta \lambda$ for each segment of length $\lambda$ is of order $l_{P}\left(\lambda / l_{P}\right)^{1 / 2}$ and takes on \pm sign with equal probability. (In the terminology used in Amelino-Camelia et al. 2002, this is referred to as a "non-systematic" effect of quantum gravity.) To simplify this part of the argument, let us assume that $\delta \lambda$ takes on only two values, viz. $\pm l_{P}\left(\lambda / l_{P}\right)^{1 / 2}$ (instead of, say, a Gaussian distribution about zero, which is more likely). If the fluctuations from the different segments were all of the same sign (i.e., coherent), then together they would contribute $\pm l_{P}\left(\lambda / l_{P}\right)^{1 / 2} \times(L / \lambda)$ to $\delta L$. But both these two cases, yielding a linear $L$-dependence for $\delta L$, are ruled out for incoherent quantum gravity fluctuations. Each has a probablity of $1 / 2^{L / \lambda} \ll 1$ for $(L / \lambda) \gg 1$. (We note that $(L / \lambda) \sim 10^{30}$ for the example involving the active galaxy PKS1413+135 considered below.) Clearly we have here a one-dimensional random walk involving $L / \lambda$ steps of equal size $(\delta \lambda)$, each step moving right or left (corresponding to + or - sign) with equal probability. The result is well-known: the cumulative fluctuation is given by $\delta L \sim \delta \lambda \times(L / \lambda)^{1 / 2}$ which is $l_{P}\left(L / l_{P}\right)^{1 / 2}$ as expected for consistency.

Alternatively, we can derive this cumulative factor $(L / \lambda)^{1 / 2}$ for the random walk model of quantum gravity by simply using the expressions for $\delta \lambda$ and $\delta L$ themselves. The cumulative factor $\mathcal{C}_{\alpha}$ defined by

$$
\delta L=\mathcal{C}_{\alpha} \delta \lambda
$$

is given, for the random walk $(\alpha=1 / 2)$ case, by

$$
\mathcal{C}_{\alpha=1 / 2}=\frac{\delta L}{\delta \lambda}=\left(\frac{L}{\lambda}\right)^{\frac{1}{2}}
$$

since $\delta L \sim l_{P}\left(L / l_{P}\right)^{1 / 2}$ and $\delta \lambda \sim l_{P}\left(\lambda / l_{P}\right)^{1 / 2}$.
The result is as expected.

But while we have some intuitive understanding of the cumulative factor $\mathcal{C}_{\alpha=1 / 2}$ for the random walk model, viz., why it goes as the square-root of the number $L / \lambda$ of "steps", we have much less intuition for the other cases. Nevertheless, since we know $\delta L$ and $\delta \lambda$, we can use Eq. (1) to find the cumulative factors, with the results

$$
\mathcal{C}_{\alpha}=\left(\frac{L}{\lambda}\right)^{1-\alpha}
$$

in particular,

$$
\mathcal{C}_{\alpha=2 / 3}=(L / \lambda)^{1 / 3}, \quad \mathcal{C}_{\alpha=1}=(L / \lambda)^{0}=1,
$$

for the holographic, $\alpha=2 / 3$, case and the "standard", $\alpha=1$, case respectively. Note that $\mathcal{C}_{\alpha=1}=$ 1 is independent of $L$. Strange as it may seem, the result is not unreasonable if we recall, for this "standard" model, $\delta l \sim l_{P}$, independent of $l$. The crucial point to remember is that, for the three quantum gravity models under consideration, none of the cumulative factors is linear in $(L / \lambda)$. (In fact, according to Eq. (3), the cumulative effects are linear in $L / \lambda$ only for the physically unacceptable case of $\alpha=0$ for which $\delta l \sim l$.) To obtain the correct cumulative factor (given by Eq. (3)) from what we may inadvertently think it is, viz., $(L / \lambda)$ (independent of $\alpha$ ), we have to put in the correction factor $(\lambda / L)^{\alpha}$.

With the correct cumulative factors for the various quantum gravity models at hand, we can now examine the prospect of probing Planck-scale physics by observing very distant sources. Consider the phase behavior of radiation with wavelength $\lambda$ received from a celestial source located at a distance $L$ away. Fundamentally, the wavelength defines the minimum length scale over which physical quantities such as phase and group velocities (and hence dispersion relations) can be defined. Thus, the uncertainty in $\lambda$ introduced by spacetime foam is the starting point for our analysis. A wave will travel a distance equal to its own wavelength $\lambda$ in a time $t=\lambda / v_{g}$ where $v_{g}$ is the group velocity of propagation, and the phase of the wave consequently changes by an amount

$$
\phi=2 \pi \frac{v_{p} t}{\lambda}=2 \pi \frac{v_{p}}{v_{g}},
$$

(i.e., if $v_{p}=v_{g}, \phi=2 \pi$ ) where $v_{p}$ is the phase velocity of the light wave. Quantum gravity fluc- 
tuations, however, introduce random uncertainties into this phase which is simply

$$
\delta \phi=2 \pi \delta\left(\frac{v_{p}}{v_{g}}\right) .
$$

We now argue that, due to quantum fluctuations of energy-momentum, the standard radiation dispersion relation $E^{2}-c^{2} p^{2}=0$ should be changed to $E^{2}-c^{2} p^{2} \sim E^{2}\left(E / E_{P}\right)^{\alpha}$. Recalling that $v_{p}=E / p$ and $v_{g}=d E / d p$, we obtain

$$
\delta\left(\frac{v_{p}}{v_{g}}\right) \sim \pm\left(\frac{E}{E_{P}}\right)^{\alpha}= \pm\left(\frac{l_{P}}{\lambda}\right)^{\alpha}
$$

where we have used $E / E_{P}=l_{P} / \lambda$. We emphasize that this may be either an incremental advance or a retardation in the phase.

In travelling over the macroscopically large distance, $L$, from source to observer an electromagnetic wave is continually subjected to random, incoherent spacetime fluctuations. Therefore, by our previous argument, the cumulative statistical phase dispersion is $\Delta \phi=\mathcal{C}_{\alpha} \delta \phi$, that is

$$
\Delta \phi=2 \pi a\left(\frac{l_{P}}{\lambda}\right)^{\alpha}\left(\frac{L}{\lambda}\right)^{1-\alpha}=2 \pi a \frac{l_{P}^{\alpha} L^{1-\alpha}}{\lambda}
$$

where $a \sim 1$. This, we believe, is the fundamental error in the Lieu \& Hillman paper, where they assume that the microscale fluctuations induced by quantum gravity into the phase of electromagnetic waves are coherently magnified by the factor $L / \lambda$ (see their equation (11)) rather than $(L / \lambda)^{1-\alpha}$.

In stellar interferometry, following Lieu and Hillman's(Lieu \& Hillman 2002, 2003) reasoning, for light waves from an astronomical source incident upon a two element interferometer to subsequently form interference fringes, it is necessary that $\Delta \phi \lesssim 2 \pi$. But the analysis of the principles of interferometry of distant incoherent astronomical "point" sources can be tricky. The local spatial coherence across an interferometer's aperature for photons from a distant point source (i.e., plane waves) is a reflection of the fact that all photons have the same resultant phase differences across the interferometer. However, this local coherence can be lost if there is an intervening medium such as a turbulent plasma or spacetime foam capable of introducing small changes into the "effective" phases of the photon stream falling on the interferometer. Such spacetime foam-induced phase differences are themselves incoherent and therefore must be treated with the correct cumulative factors $\mathcal{C}_{\alpha}$ appropriate for the quantum gravity model under consideration.

Fluctuations due to quantum gravity are very minuscule, so they can be detected only if there is a huge cumulative effect from "summing" up the individual fluctuations. But since the cumulative factor for the "standard" model of quantum gravity (for which $\alpha=1$ ) is 1, i.e., there is no cumulative effect, obviously the proposed approach (of applying spacetime fluctuations on the phase coherence of light from extragalactic sources to probe the graininess of spacetime) cannot be used to rule out (or confirm) the $\alpha=1$ model. This is the first result of this Letter.

To rule out models with $\alpha<1$, the strategy is to to look for interference fringes for which the phase coherence of light from the distant sources should have been lost (i.e., $\Delta \phi \gtrsim 2 \pi$ ) for that value of $\alpha$ according to theoretical calculations. Consider the example cited by Lieu and Hillman(Lieu \& Hillman 2003), involving the clearly visible Airy rings in an observation of the active galaxy $\operatorname{PKS} 1413+135(L=1.216 \mathrm{Gpc})$ by the HST at $\lambda=1.6 \mu \mathrm{m}$ wavelength(Perlman et al. 2002). For this example, Eq. (8) yields $\Delta \phi \sim 10 \times 2 \pi a$ for the random walk $\alpha=1 / 2$ model and $\Delta \phi \sim 10^{-9} \times 2 \pi a$ for the holography $\alpha=2 / 3$ model. Since we expect $a \sim 1$, the observation of Airy rings in this case would seem to marginally rule out the random walk model. (But of course proponents of the random walk model can equally claim that their favorite model is still marginally acceptable.) On the other hand, the holography model is obviously not ruled out. This finding contradicts the conclusion reached recently by Lieu and Hillman(Lieu \& Hillman 2003) who argued that the HST detection of Airy rings from PKS1413+135 has ruled out a majority of modern models of quantum gravity, including the "standard" $\alpha=1$ model. (Earlier, Lieu and Hillman(Lieu \& Hillman 2002) had claimed to have ruled out the $\alpha=2 / 3$ model by noticing that interference effects were clearly seen in the Infra-red Optical Telescope Array(van Belle et al. 2002) at $\lambda=2.2 \mu \mathrm{m}$ light from the star $\mathrm{S}$ Ser which is $\sim 1$ $\mathrm{kpc}$ away.) The resolution of this disagreement lies in the fact that Lieu and Hillman neglected to take into account the correction factor in estimating the cumulative effects of spacetime foam. This 
neglect resulted in their overestimate of the cumulative effects by a factor $(L / \lambda)^{\alpha}$ : for the case of PKS1413+135, $10^{15}$ for $\alpha=1 / 2,10^{20}$ for $\alpha=2 / 3$, and $10^{30}$ for $\alpha=1$ respectively. Subsequent work by Ragazzoni, Turatto, and Gaessler(Ragazzoni et al. 2003) contains the same error of assuming that the cumulative factor is $(L / \lambda)$ rather than the correct factor $(L / \lambda)^{1-\alpha}$. Their claim that the $\alpha=2 / 3$ model and the $\alpha=1$ model are ruled out is also incorrect. We note that Coule(Coule 2003) has independently pointed out that "Planck scale is still safe from stellar images" using another argument.

Now consider a delta function type pulse of radiation from a source at a distance $L$. This pulse will spread in time because of quantum gravity fluctuations and the overall time dispersion in the pulse can be simply related to the aforementioned phase dispersion, i.e.,

$$
\Delta T \sim \Delta \phi\left(\frac{\lambda}{2 \pi v_{p}}\right)=\left(\frac{l_{P}}{L}\right)^{\alpha} \frac{L}{v_{p}} .
$$

The width of the pulse increases with distance as $L^{1-\alpha}$ but is independent of the wavelength (i.e., it is not dispersive in frequency space). For example, consider gamma ray bursts at a cosmological distance $L \sim 10^{28} \mathrm{~cm}$. Then the overall time dispersion in the pulse is only given by an unobservably small $\Delta T \sim 10^{17} \times\left(10^{-61}\right)^{\alpha}$ sec. Thus GB's also do not offer a promising venue for testing for quantum foam, even at high Z's.

In conclusion, we have examined the possibility of using spacetime foam-induced phase incoherence of light from distant galaxies and gammaray bursters to probe Planck-scale physics. These effects are real and are magnified over the large distances traversed by radiation from distant extragalactic sources. However, the effects of spacetime foam are incoherent and do not grow linearly with distance, instead increasing as $L^{1-\alpha}$. As a result, the cumulative effects of spacetime fluctuations on the phase coherence of light are too small to be observable. Therefore, we refute the recent claims(Lieu \& Hillman 2003; Ragazzoni et al. 2003) that modern theories of quantum gravity have been observationally ruled out.

This work was supported in part by the US Department of Energy and by the Bahnson Fund at
UNC. YJN thanks D.H. Coule, E.S. Perlman, and G.T. van Belle for useful email correspondence.

\section{REFERENCES}

Aloisio, R., Blasi, P., Galante, A., Ghia, P. L., \& Grillo, A. F. 2002, arXiv:astro-ph/0205271

Amelino-Camelia, G. 1994, Mod. Phys. Lett. A9, 3415

Amelino-Camelia, G. \& Piran, T. 2001, Phys. Lett. B497, 265

Amelino-Camelia, G., Ng, Y. J., \& van Dam, H. 2002, arXiv: gr-qc/0204077

Coule, D. H. 2003, arXiv:astro-ph/0302333

Lieu, R., \& Hillman, L. W. 2002, arXiv:astro$\mathrm{ph} / 0211402$

Lieu, R., \& Hillman, L. W. 2003, arXiv:astroph/0301184 (accepted for publication in ApJL).

Misner, C. W., Thorne, K. S., \& Wheeler, J. A. 1973, Gravitation (San Francisco: Freeman), 1190

Ng, Y. J., \& van Dam, H. 1994, Mod. Phys. Lett. A9, 335

Ng, Y. J., \& van Dam, H. 2000, Found. Phys. 30, 795

Ng, Y. J. 2001, Phys. Rev. Lett. 86, 2946; (erratum) 88,139902

Ng, Y. J., Lee, D. S., Oh, M. C., \& van Dam, H. 2001, Phys. Lett. B507, 236

Perlman, E. S. et al. 2002, AJ 124, 2401

Ragazzoni, R., Turatto, M., \& Gaessler, W. 2003, arXiv:astro-ph/0303043 (accepted for publication in ApJL)

Susskind, L. 1995, J. Math. Phys. 36, 6377

'tHooft, G. 1993, in Salamfestschrift, ed. A. Ali et al. (Singapore: World Scientific), 284

van Belle, G. T., Thompson, R. R., \& CreechEakman, M. J. 2002, AJ 124, 1706

This 2-column preprint was prepared with the AAS LATEX macros v5.0. 